\title{
Perfil das mulheres usuárias de cocaína e crack atendidas em Centro de Atenção Psicossocial
}

\author{
Profile of women crack cocaine users attending a Psychosocial Care Center \\ Perfil de las mujeres usuarias de cocaína y crack asistidas en Centro de Atención Psicosocial
}

\author{
Érika Barbosa de Oliveira Silval, Adriana Lenho de Figueiredo PereiralI
}

\begin{abstract}
RESUMO: Estudo exploratório e documental, que objetivou descrever o perfil social e clínico das mulheres usuárias de cocaína e crack e identificar as características do cuidado prestado às mulheres em Centro de Atenção Psicossocial (CAPS ad). O estudo foi realizado no único CAPS ad do município de Duque de Caxias, Estado do Rio de Janeiro, em 2014. Foram analisados 113 prontuários de mulheres em tratamento nesse serviço público de saúde. Os dados receberam tratamento estatístico descritivo. As usuárias de cocaína e crack são predominantemente solteiras, com filhos menores de idade, sem ocupação remunerada, que apresentam alterações psicóticas ou do humor e utilizam outras drogas concomitantemente ao uso destas substâncias. O cuidado psicossocial está direcionado para os problemas psíquicos, sociais e clínicos das mulheres, mas com ênfase nas ações individuais, no tratamento psiquiátrico e fortalecimento da rede social de apoio. Recomenda-se abordagem terapêutica mais sensível para as questões de gênero dessa clientela.

Palavras-Chave: Saúde da mulher; gênero e saúde; cocaína, crack; saúde mental.
\end{abstract}

ABSTRACT: This exploratory, documentary study to describe the social and clinical profile of women users of cocaine and crack, and to characterize the psychosocial care provided to women at a psychosocial care center, was conducted at the only such center in Duque de Caxias, Rio de Janeiro State, in 2014. The medical records of 113 women treated at this public health service were examined, and the resulting data received descriptive statistical treatment. The cocaine and crack users were predominantly single with juvenile children, no paid employment, presented psychotic or mood disorders, and used other drugs besides cocaine and crack. Psychosocial care was directed to the women's psychic, social and clinical problems, but with the emphasis on individual actions, psychiatric treatment and strengthening the social support network. It is recommended that the therapeutic approach be made more sensitive to gender issues in this clientele.

Keywords: Women's health; gender and health; crack cocaine; mental health.

RESUMEN: Investigación exploratoria y documental, cuyo objetivo fue describir el perfil social y clínico de las usuarias de cocaína y crack e identificar las características del cuidado prestado a las mujeres en Centro de Atención Psicosocial (CAPS). La investigación se realizó en el único CAPS del municipio de Duque de Caxias, Río de Janeiro, en 2014, donde se han analizado 113 registros de historias clínicas de las mujeres en tratamiento. Los datos recibieron tratamiento estadístico descriptivo. Estas usuarias de cocaína e crack son predominantemente solteras, tienen hijos menores de edad, están desempleadas, presentan trastornos psicóticos o del estado de ánimo y consumen otras drogas concomitantemente el uso de estas sustancias. El cuidado psicosocial se dirige a los problemas psíquicos, sociales y clínicos de las mujeres, con énfasis en las acciones individuales, en tratamiento psiquiátrico y fortalecimiento de la red de apoyo social. Se recomiendan un abordaje terapéutico más sensible para las cuestiones de género de esta clientela.

Palabras Clave: Salud de la mujer; género y salud; cocaína, crack; salud mental.

\section{INTRODUÇÃO}

O consumo de drogas atinge a sociedade de forma heterogênea e causa repercussões diversas para saúde das populações. Apesar do uso de cocaína e seus derivados, como o crack e a merla, ter diminuído ou se mantido estável na maioria dos países sul-americanos, no Brasil houve um aumento da prevalência do consumo dessa substância, estimada em 1,75\% na população em geral em $2011^{1}$.

Inquérito epidemiológico brasileiro revela que as mulheres são minoria entre os usuários de cocaína e crack, representando cerca de 40\% dos usuários. Ao considerar os dados referentes ao uso de crack e/ou similares em 2012, houve predomínio dos usuários do sexo masculino, que correspondem a $78,7 \%$ da totalidade. Estes dados se referem ao consumo dessas substâncias nos espaços públicos ou cenas abertas de uso, tais como cracolândias, casas abandonadas, clínicas e no sistema prisional, e não em domicílios privados ${ }^{2}$.

Em decorrência desse perfil epidemiológico, as distinções do gênero feminino na problemática do

\footnotetext{
${ }^{I}$ Mestre em Enfermagem pela Faculdade de Enfermagem da Universidade do Estado do Rio de Janeiro. Professora Assistente na Universidade do Grande Rio. Professora Substituta na Escola de Enfermagem Anna Nery. Rio de Janeiro, Brasil. E-mail: erikabos.enf@gmail.com

IIProfessora Adjunta do Departamento de Enfermagem Materno-Infantil e do Programa de Pós-Graduação em Enfermagem, Universidade do Estado do Rio de Janeiro. Brasil. E-mail: adrianalenho.uerj@gmail.com.
} 
consumo de cocaína e crack não têm sido destacadas nas políticas públicas e nas ações de saúde. Os estudos sobre o consumo de crack sob a perspectiva de gênero ainda são incipientes ${ }^{3}$.

A articulação entre o gênero e a saúde mental é proposta pela Política Nacional de Assistência Integral a Mulher (PNAISM), considerando que os aspectos da vida cotidiana das mulheres e a sobrecarga das responsabilidades por elas assumidas podem prejudicar o estado de bem-estar psíquico ${ }^{4}$. As questões do gênero feminino participam na complexidade do fenômeno do uso de drogas produzindo desafios para o cuidado às usuárias de cocaína e crack.

Os Centros de Atenção Psicossocial para Álcool e Drogas (CAPS ad) são serviços especializados no cuidado para demandas decorrentes do uso dessas substâncias, e devem prestar cuidado por equipe multidisciplinar, buscando a compreensão da realidade sociocultural dessas usuárias para dar respostas efetivas às demandas específicas do gênero feminino.

Tendo em vista as especificidades do gênero feminino no enfrentamento dos problemas relacionados ao uso de cocaína e crack, foi proposto o presente estudo que objetivou descrever o perfil social e clínico das mulheres usuárias de cocaína e crack e identificar as características do cuidado prestado às mulheres no CAPS ad.

\section{ReVisÃo DE LITERATURA}

As mulheres apresentam vulnerabilidades relacionadas às questões de gênero, que as expõem a padrões de sofrimento, adoecimento e morte distintos dos homens. Portanto, a construção do cuidado integral à saúde das mulheres requer o estabelecimento de uma comunicação transversal entre diversas áreas de conhecimento. $\mathrm{O}$ cuidado transversal abrange a produção de saúde e de subjetividade, compreendendo os fenômenos complexos que envolvem o sujeito em seu contexto de vida ${ }^{4,5}$.

Com o movimento da Reforma Psiquiátrica, o sistema de saúde brasileiro organizou a rede de assistência centrada na atenção comunitária, com ênfase na prevenção do adoecimento psíquico, reabilitação psicossocial e reinserção social dos indivíduos. Nesse contexto, a oferta de cuidados às pessoas que consomem o álcool e outras drogas foi priorizada em locais extra-hospitalares e interligados à rede de serviços de saúde ${ }^{6}$.

Os CAPS ad são serviços de atenção diária e buscam promover as potencialidades para a produção de saúde por meio do cuidado centrado nas necessidades das pessoas ${ }^{7,8}$. Esse serviço conta com a atuação de uma equipe de saúde multidisciplinar, que inclui os profissionais de enfermagem. Esta equipe tem a responsabilidade de elaborar, em conjunto com o usuário e seus familiares, um projeto terapêutico singular e de acordo com o contexto cotidiano dos indivíduos 9 .
Na construção do projeto terapêutico, recomenda-se considerar as especificidades do gênero feminino. Por mais que homens e mulheres partilhem experiências de saúde-doença semelhantes, as mulheres as vivenciam de forma diferente por conta da socialização distintiva mediada pelo gênero ${ }^{10}$.

C cuidado psicossocial singular considera o sujeito e cidadão dentro de sua história de vida, em seu contexto econômico, social e cultural, e sua estrutura psicológica. Gênero é um constructo sociocultural ${ }^{4}$, portanto é imprescindível na abordagem psicossocial. Assim, conhecer as especificidades do gênero feminino entre os usuários de cocaína e crack contribui para o conhecimento das diferenças existentes entre homens e mulheres no enfrentamento do uso de substâncias e é imprescindível para estabelecer um cuidado equitativo, integral e efetivo para as mulheres.

\section{Metodologia}

Trata-se de um estudo exploratório e documental, realizado no único CAPS ad do município de Duque de Caxias, situado na Baixada Fluminense, região metropolitana do Estado do Rio de Janeiro. Este município tenha uma população estimada de 873.921 residentes, em 2013, e apresenta índice de médio desenvolvimento humano $(0,753)^{11}$.

A partir da totalidade de prontuários da clientela ativa no serviço, foram destacados os prontuários das mulheres em situação de drogadição e, posteriormente, foram selecionados os prontuários das mulheres usuárias de crack e cocaína. Portanto, foram incluídos no estudo os dados registrados das usuárias dessas substâncias e que estavam em tratamento regular no serviço, sendo excluídos os prontuários inativos, com registro de abandono do tratamento ou de referência para outro serviço de saúde.

As variáveis do estudo foram os dados sociais das mulheres (idade, estado civil, grau de instrução, situação de moradia, existência de prole, situação de moradia, tipo de ocupação, fonte de renda e envolvimento com a justiça); os dados clínicos (admissão no serviço; participação do familiar no tratamento; agravo à saúde relacionado ao consumo da droga; tipo de substância de primeiro uso e atualmente consumida; idade inicial de uso de substâncias e de crack/cocaína) e os dados do cuidado psicossocial (para os problemas sociais, transtornos psíquicos, enfrentamento da dependência e saúde da mulher).

A coleta de dados foi realizada no mês de maio de 2014, sendo utilizado roteiro de análise documental estruturado. As informações coletadas foram armazenadas em banco de dados informatizado em Excel do Microsoft Office 2003. Posteriormente, foram tabulados e submetidos à estatística descritiva. A análise considerou as frequências absolutas e percentuais, confrontadas com a literatura científica sobre a temática investigada. 
O estudo observou os princípios éticos e obteve parecer favorável da Comissão de Ética em Pesquisa da Universidade do Estado do Rio de Janeiro (COEP/ UERJ) nº 628.205.

\section{Resultados e Discussão}

Entre os 361 prontuários da clientela ativa no serviço, verificou-se que 158 prontuários eram de mulheres em tratamento no CAPS ad. Deste quantitativo, 113 (100\%) prontuários pertenciam às mulheres usuárias de cocaína e crack atendidas na instituição.

A partir da análise dos prontuários dessas usuárias, identificou-se o predomínio das mulheres adultas com idade acima de 30 anos (52,2\%), seguidas daquelas com idade de 20 a 29 anos (38\%). A maior parte delas é solteira $(55,7 \%)$ com prole menor de idade $(55,8 \%)$, que reside com os familiares $(62,4 \%)$. Destacam-se as que não tem ocupação laboral remunerada $(47,8 \%)$ e dependentes da renda da família para o seu sustento (39\%), como pode ser observado na Tabela 1 .

Os homens são os principais usuários de cocaína e crack em espaços públicos de consumo no Brasil, com a proporção de $78,7 \%$, bem como entre clientela atendida em CAPS $a^{2}$. Pesquisa nesse tipo de serviço na Região Sul do país encontrou o percentual de 82,1\% para os usuários do sexo masculino ${ }^{12}$. As mulheres em tratamento no CAPS ad em que foi desenvolvido o presente estudo representam 43,8\% do total da clientela. Considerando apenas as usuárias de cocaína e crack, este grupo correspondeu a 31,3\% dos 361 prontuários ativos no serviço. Esta maior representatividade feminina pode denotar uma especificidade

TABELA 1: Perfil sociodemográfico das usuárias de crack e cocaína em tratamento no CAPS ad. Município de Duque de Caxias, Rio de Janeiro, maio, 2014. (N=113)

\begin{tabular}{|c|c|c|}
\hline Variável & $f$ & $\%$ \\
\hline \multicolumn{3}{|l|}{ Faixa etária } \\
\hline Abaixo de 15 anos & 3 & 2,6 \\
\hline 15 a 19 anos & 8 & 7,2 \\
\hline 20 a 29 anos & 43 & 38 \\
\hline Acima de 30 anos & 59 & 52,2 \\
\hline \multicolumn{3}{|l|}{ Situação conjugal } \\
\hline Solteira & 63 & 55,7 \\
\hline Casada/União estável & 35 & 31,1 \\
\hline Divorciada/ Separada & 13 & 11,5 \\
\hline Viúva & 2 & 1,7 \\
\hline \multicolumn{3}{|l|}{ Moradia e habitação } \\
\hline Mora com famíliares & 71 & 62,8 \\
\hline Mora com cônjuge/parceiro & 28 & 24,7 \\
\hline Mora sozinha & 2 & 1,7 \\
\hline Em situação de rua & 8 & 7,1 \\
\hline Abrigos/Albergues/instituições & 4 & 3,5 \\
\hline \multicolumn{3}{|l|}{ Ocupação } \\
\hline Estudante & 6 & 5,3 \\
\hline Emprego formal & 11 & 9,7 \\
\hline Emprego informal & 17 & 15 \\
\hline Dona-de-casa/do lar & 19 & 16,8 \\
\hline Sem ocupação & 54 & 47,8 \\
\hline Sem informação no prontuário & 6 & 5,3 \\
\hline \multicolumn{3}{|l|}{ Outra fonte de renda além da ocupação principal } \\
\hline Pensão por morte & 2 & 1,7 \\
\hline Pedinte na rua & 2 & 1,7 \\
\hline Bolsa Família & 4 & 3,5 \\
\hline Envolvimento com a criminalidade (furtos, tráfico) & 6 & 5,3 \\
\hline Prostituição & 14 & 12,4 \\
\hline Sustento familiar & 44 & 39,0 \\
\hline \multicolumn{3}{|l|}{ Existência de prole } \\
\hline Sim, Menor de idade & 63 & 55,8 \\
\hline Sim, Maior de idade & 20 & 17,7 \\
\hline Não & 37 & 34,0 \\
\hline
\end{tabular}

Fonte: Prontuários das usuárias. Arquivo do serviço do CAPS ad. 
da distribuição por sexo da população de usuários de cocaína e crack no município de Duque de Caxias, como também suscitar que as questões relacionadas ao gênero estão envolvidas.

A variável sexo não revela apenas um dado epidemiológico, mas sugere que as relações de gênero em saúde mental precisam ser consideradas no projetos terapêuticos. $\mathrm{O}$ gênero é estruturante das relações de poder e determina o processo de saúde-doença ${ }^{4}$. Portanto, é necessário perceber a realidade social do sujeito do cuidado na perspectiva do gênero.

$\mathrm{O}$ aumento dos indicadores do uso de drogas psicoativas entre mulheres foi encontrado em estudos brasileiros, sendo um desafio para a saúde pública e os serviços especializados ${ }^{13}$. A construção dos projetos terapêuticos deve considerar as necessidades dessas mulheres, que são solteiras, mães, em idade fértil, sem ocupação laboral, renda própria e, por vezes, podem estar envolvidas em situações de violência e prostituição como forma de acesso à droga. Portanto, estratégias terapêuticas devem incluir o acesso à qualificação para o mercado de trabalho, aquisição de renda própria, manutenção dos cuidados e guarda dos filhos menores, entre outras.

A prevalência de mulheres solteiras, com nível de escolaridade baixo, entre a $4^{\mathrm{a}}$ e a $8^{\mathrm{a}}$ série do nível fundamental são características observadas no perfil de usuárias dessas substâncias no país ${ }^{2}$, o que dificulta o acesso ao trabalho e à aquisição de renda. Na presente pesquisa, não foi possível identificar este dado em virtude de sua ausência em 52,2\% dos prontuários analisados.

Considerando a ocupação e a renda, estudos verificaram que os dependentes de crack e seus similares apresentam situação de desemprego e são provenientes de famílias desestruturadas e de baixa renda ${ }^{3}$. Este status econômico também foi observado nos resultados aqui apresentados, visto que em 69,9\% dos prontuários das mulheres usuárias de cocaína e crack registravam sem ocupação remunerada.

O uso de substâncias por mulheres está relacionado ao contexto cultural, social, familiar e individual, que envolve fatores de risco, como o uso do corpo para obter droga, a prostituição e as doenças sexualmente transmissíveis ${ }^{13}$. Nos prontuários analisados, 12,4\% das mulheres estavam envolvidas com prostituição e 5,3\% com criminalidade. No entanto, o elevado percentual de mulheres sem renda própria e que dependem do sustento familiar suscita a necessidade de compreender como estas mulheres adquirem a droga e que implicações isto tem sobre suas vidas. Investigar as formas de aquisição de drogas é importante para a preservação da saúde e da integridade psicossocial, tendo em vista que estudos verificaram que uma parcela considerável das usuárias tem envolvimento com práticas de roubos, furtos, assaltos, situação de rua, prostituição e troca de sexo por droga 2,13 .
Na análise das características clínicas das usuárias de cocaína e crack, identificou-se que o acesso das mulheres ao tratamento no CAPS ad ocorreu principalmente por referência de outro serviço de saúde - 42 $(37,2 \%)$ e por indicação de amigo ou familiar - 22 $(17,7 \%)$. Cabe destacar que este dado estava ausente em 22 (19,5\%) dos prontuários pesquisados.

Quanto à participação da família no tratamento, foram 86 (76\%) prontuários com registro afirmativo do apoio familiar no processo terapêutico. Em 82 (75,6\%) dos prontuários havia registro de outros agravos à saúde relacionados ao uso de cocaína e crack, com destaque para as alterações psicóticas - 48 (42,5\%) e do humor - $23(20,3 \%)$.

Sobre a incidência de outros transtornos mentais associados ao uso de substâncias, estima-se de 6 a 8\% da população ${ }^{14}$. $\mathrm{O}$ crack, além de elevar o potencial de sintomas depressivos, ansiosos e de outras comorbidades, também causa menos motivação para a mudança e menor adesão ao tratamento ${ }^{13}$.

Em quase a totalidade dos prontuários - 111 $(98,3 \%)$ - foi registrado o consumo de outras drogas concomitantemente ao uso de cocaína e crack, com predomínio do tabaco (77\%), maconha (63\%) e álcool $(45,1 \%)$. O consumo de múltiplas drogas, em um curto período de tempo, e simultaneamente, tem sido observado no atual padrão compulsivo do uso dessas substâncias, substituindo paulatinamente o uso exclusivo $^{15}$. A nicotina, álcool e maconha são drogas mais associadas à cocaína e ao crack no país ${ }^{12}$.

$\bigcirc$ poliuso de substâncias tem início nos jovens com menos de 19 anos de idade, o revela a importância das ações preventivas na adolescência. As primeiras experiências com drogas ocorrem em sua maioria nessa fase da vida, momento em que os indivíduos estão mais vulneráveis, o que requer a identificação dos fatores socioculturais e psicológicos predisponentes para o uso abusivo de substâncias ${ }^{16}$.

$\mathrm{Na}$ busca de seu papel, dentro do seu circulo social, o adolescente pode ficar exposto à drogadição, seja por estímulos de colegas, familiares, apelo publicitário, ou até mesmo pela curiosidade ${ }^{17}$. Apesar disso, durante a formação do adolescente há fatores que podem ser protetores para o uso de substâncias, como o fortalecimento de vínculos afetivos na família, envolvimento com atividade escolar, acesso às informações sobre o uso de drogas, entre outros ${ }^{13}$.

Considerando a idade e o tipo de droga de primeiro uso, o período da adolescência foi o momento de início da utilização de substâncias psicoativas, com predomínio das faixas etárias abaixo de 15 anos - 49 $(43,4 \%)$ e entre 15 e 19 anos - 34 (30\%). A maconha foi a principal droga envolvida no primeiro consumo $41(36,3 \%)$, seguida do álcool 21 (18,6\%). Esse período da vida também foi aquele em que a maior parte das mulheres iniciou o consumo de cocaína e crack, como pode ser observado na Tabela 2. 
TABELA 2: Perfil Clínico das usuárias de crack e cocaína em tratamento no CAPS ad. Município de Duque de Caxias, Rio de Janeiro, maio de 2014. (N=113)

\begin{tabular}{lcc}
\hline Variável & $f$ & $\%$ \\
\hline Idade inicial de uso de substâncias & 49 & 43,4 \\
Abaixo de 15 anos & 34 & 30 \\
15 a 19 anos & 11 & 9,7 \\
20 a 29 anos & 1 & 0,9 \\
A partir de 30 anos & 18 & 16 \\
Sem informação no prontuário & & \\
Substância de primeiro uso & 1 & 0,9 \\
Drogas sintéticas (LSD, Ecstazy) & 3 & 2,6 \\
Inalantes e Solventes (Loló, Lança-perfume) & 11 & 9,7 \\
Cocaína & 13 & 11,5 \\
Tabaco & 21 & 18,6 \\
Álcool & 41 & 36,3 \\
Maconha & 23 & 20,3 \\
Sem informação no prontuário & & \\
Idade inicial do uso de crack/cocaína & 23 & 20,3 \\
Abaixo de 15 anos & 27 & 23,9 \\
15 a 19 anos & 24 & 21,2 \\
20 a 29 anos & 10 & 8,8 \\
A partir de 30 anos & 18 & 16 \\
Sem informação no prontuário & & \\
Associação com outras drogas & 2 & 1,7 \\
Não & 111 & 98,3 \\
Sim & 1 & 0,9 \\
$\quad$ Droga Injetável & 47 & 41,6 \\
$\quad$ Drogas sintéticas (LSD, Ecstasy) & 50 & 44,3 \\
$\quad$ Inalantes e Solventes (Loló, Lança-perfume) & 51 & 45,1 \\
$\quad$ Álcool & 71 & 63 \\
$\quad$ Maconha & 87 & 77 \\
Outro agravo a saúde relacionada ao uso do crack/cocaína & & \\
Não & 31 & 27,4 \\
Sim & 82 & 75,6 \\
$\quad$ Alterações Psicóticas & 48 & 42,5 \\
$\quad$ Alterações do Humor & 23 & 20,3 \\
$\quad$ Dlterações Comportamentais & 18 & 16 \\
\hline
\end{tabular}

Fonte: Prontuários das usuárias. Arquivo do serviço do CAPS ad.

Sobre os cuidados descritos nos prontuários, os atendimentos individuais com os profissionais de saúde foram preponderantes no plano terapêutico das usuárias, destacando-se as ações para os problemas psíquicos, como acompanhamento com psiquiatra 112 (99,1\%); sociais, como acompanhamento com assistente social - 94 (83,2\%); e clínicos, como acompanhamento com neurologista - $16(14,2 \%)$, conforme a Tabela 3. Outras estratégias de cuidado também foram adotadas, como o fortalecimento da rede social de apoio (69\%), a prescrição de psicotrópicos (69\%) para transtornos psíquicos e, em menor proporção e a participação em grupo de mulheres (44,3\%).

Durante a análise documental, constatou-se que os planos de cuidado ou projetos terapêuticos não es- tavam explicitamente descritos nos prontuários. Foram encontrados registros de ações voltadas para a resolução dos problemas sociais, de recuperação da cidadania e fortalecimento da rede social de apoio de cada usuária. Os cuidados registrados também denotam a primazia nas ações individualizadas, ao invés de uma construção coletiva e multidisciplinar. Apesar do cuidado multidisciplinar não excluir os atendimentos e consultas individuais, ele precisa ser construído coletivamente para a ampliação do conhecimento da pessoa a ser cuidada, do seu contexto sociocultural e de sua relação com a droga.

Os atendimentos oferecidos pelo CAPS ad devem incluir a consulta individual, atendimento em grupo, oficinas terapêuticas, visitas domiciliares, repouso e desintoxicação ambulatorial, além de es- 
TABEIA 3: Cuidados descritos no plano terapêutico individual das mulheres usuárias de crack/ cocaína do CAPS ad. Município de Duque de Caxias, Rio de Janeiro, maio, 2014. (N=113)

\begin{tabular}{|c|c|c|}
\hline Variáveis & $f$ & $\%$ \\
\hline \multicolumn{3}{|l|}{ Cuidados para os problemas sociais } \\
\hline Resgate de documentação civil & 1 & 0,9 \\
\hline Indicação para capacitação profissional & 2 & 1,7 \\
\hline Interlocução com Ministério Público & 2 & 1,7 \\
\hline Orientações trabalhistas e sobre direitos previdenciários & 3 & 2,6 \\
\hline Interlocução com Vara da Infância e Conselho Tutelar & 7 & 6,2 \\
\hline Encaminhamento para programas de moradia e renda & 14 & 12,4 \\
\hline Acompanhamento individual com assistente social & 94 & 83,2 \\
\hline \multicolumn{3}{|l|}{ Cuidados para os transtornos psíquicos } \\
\hline Indicação de internação psiquiátrica & 8 & 7,1 \\
\hline Acompanhamento individual com a psicologia & 25 & 22,1 \\
\hline Prescrição de Psicotrópicos & 78 & 69 \\
\hline Acompanhamento individual com psiquiatra & 112 & 99,1 \\
\hline \multicolumn{3}{|l|}{ Cuidados para os problemas clínicos } \\
\hline Encaminhamento a outras especialidades fora do serviço & 10 & 8,8 \\
\hline Acompanhamento individual com neurologista no serviço & 16 & 14,2 \\
\hline Acompanhamento individual com clínico geral no serviço & 88 & 77,9 \\
\hline \multicolumn{3}{|l|}{ Cuidados para o enfrentamento da dependência } \\
\hline Interlocução com a escola & 6 & 5,3 \\
\hline Internação para desintoxicação & 9 & 8 \\
\hline Estratégias motivacionais & 16 & 14,2 \\
\hline Busca ativa de usuários que não seguem o plano terapêutico & 20 & 17,7 \\
\hline Inclusão em grupos terapêuticos diversos & 21 & 18,6 \\
\hline Plano individual com estratégias para a abstinência & 23 & 20,3 \\
\hline Terapia ocupacional & 25 & 22,1 \\
\hline Fortalecimento da rede de apoio & 78 & 69 \\
\hline \multicolumn{3}{|l|}{ Cuidados específicos para saúde da mulher } \\
\hline Encaminhamento à obstetrícia & 1 & 0,9 \\
\hline Ações voltadas para contracepção e prevenção de DST/HIV & 3 & 2,6 \\
\hline Encaminhamento à ginecologia & 15 & 13,2 \\
\hline Grupo de mulheres & 50 & 44,3 \\
\hline
\end{tabular}

Fonte: Prontuários das usuárias. Arquivo do serviço do CAPS ad.

paços de convivência ${ }^{7}$. Portanto, deve-se abordar as dimensões psíquica, emocional, sociocultural e familiar da pessoa a ser cuidada.

Os grupos terapêuticos são importantes estratégias de cuidado, porém apenas 18,6\% dos prontuários analisados registraram a participação dessas mulheres em grupos terapêuticos diversos e 44,3\% em grupo de mulheres. Os grupos terapêuticos podem ser educativos, de reflexão e de suporte, e possibilitam ao indivíduo a tomada de consciência como ser social e sujeito coparticipante do processo terapêutico ${ }^{18}$. Portanto, a baixa frequência dessas estratégias de cuidado pode implicar desfavoravelmente a qualidade e efetividade do cuidado psicossocial prestado à clientela feminina no CAPS ad investigado.

\section{Conclusão}

As mulheres usuárias de cocaína e crack do CAPS ad são predominantemente solteiras e mães com prole menor de idade, que não têm ocupação remunerada e vivem com a família. Elas também apresentam alterações psicóticas ou do humor, utilizam outras drogas concomitantemente ao uso dessas substâncias e apresentam perfil social e clínico semelhante aos estudos nacionais sobre esse grupo populacional.

O cuidado psicossocial prestado no CAPS ad está direcionado para os problemas psíquicos, sociais e clínicos das mulheres, com ênfase nas ações individuais, no tratamento psiquiátrico e no fortalecimento da rede social de apoio para o enfrentamento da dependência química.

Apesar das limitações do estudo documental, os resultados encontrados também indicam a necessidade de melhoria das anotações dos profissionais nos prontuários, em virtude do sub-registro de dados importantes para o projeto terapêutico.

É necessário o aprofundamento da investigação sobre as mulheres envolvidas com o uso abusivo de cocaína e crack, o cuidado prestado pela enfermagem e a adoção da abordagem de gênero no cuidado psicossocial devido às singularidades dessa clientela feminina. 


\section{REFERÊNCIAS}

1.Organização das Nações Unidas. Escritório das Nações Unidas Sobre Drogas e Crimes. Relatório Mundial sobre Drogas, 2013. NovaYork: ONU; 2013.

2.Bastos FI, Bertoni N, organizadores. Pesquisa Nacional sobre o Uso de Crack: quem são os usuários de crack e/ou similares do Brasil? Quantos são nas capitais brasileiras? Rio de Janeiro: ICICT/FIOCRUZ; 2014.

3.Souza RF, Padovani RC, Tucci AM. Consumo de crack a partir das perspectivas de gênero e vulnerabilidade: uma revisão sobre o fenômeno. Saúde debate. 2014; 38:379-92.

4.Ministério da Saúde (Br). Política Nacional de Atenção Integral à Saúde da Mulher: princípios e diretrizes. Brasília (DF): Editora MS; 2011.

5.Pedroso RT, Vieira MEM. Humanização das práticas de saúde: transversalizar em defesa da vida. Interface (Botucatu).2009; 13:695-700.

6.Ministério da Saúde (Br). A Política do Ministério da Saúde para a Atenção Integral a Usuários de Álcool e outras Drogas. 2aed. Brasília (DF): Editora MS; 2004.

7.Ministério da Saúde (Br).Portaria GM no 336 de 19 de Fevereiro de 2002. Brasília (DF): Gabinete Ministerial; 2002.

8.Ministério da Saúde (Br). Portaria GM nº 130 de 26 de Janeiro de 2012. Brasília: 2012.

9.Boccardo ACS, Zane FC, Rodrigues S, Mângia EF. O projeto terapêutico singular como estratégia de organização do cuidado nos serviços de saúde mental. Rev Ter Ocup Univ São Paulo. 2011; 22(1):85-92.

10.Lima HP, Braga VAB, Gubert FA. Interface entre gênero e saúde mental no discurso de alcoolistas: estudo qualitativo. Online Braz J Nurs [Internet]. 2010 [citado em 25 nov 2014];9(2). Disponível em: http:// www.objnursing.uff.br/index.php/nursing/article/view /j.1676-4285.2010.2907/665.
11.Serviço Brasileiro de Apoio a Micro e Pequenas Empresas. Informações socioeconômicas do Município de Duque de Caxias [site de Internet]. Rio de Janeiro: Sebrae; 2011. [citado em 27 nov 2014]. Disponível em: http://201.2.114.147/bds/bds.nsf/3C0F2AE4C6B74D7B 83257950005F787B/\$File/Duque\%20de\%20Caxias.pdf 12.Horta RL, Horta BL, Rosset AP, Horta Cl. Perfil dos usuários de crack que buscam atendimento em Centros de Atenção Psicossocial. Cad Saúde Pública. 2011; 27: 2263-70.

13.Lima HP, Macedo JQ, Braga VAB, Lemos AM, Silva Júnior IA. Caracterização de drogaditas atendidas em Centro de Atenção Psicossocial álcool e outras drogas: estudo documental. Online braz j nurs [Internet]. 2011 [citado em 24 nov 2014]; 10 (2). Disponível em: http://www.objnursing.uff.br/index.php/nursing/article/ view/3257. v.10. n.2, abr.-ago., 2011.

14.Ministério da Saúde (Br). Saúde Mental e Atenção Básica: o vínculo e o diálogo necessários. Brasília (DF): Ministério da Saúde; 2007.

15.Costa LLS, Navas ALGP, Oliveira CCC, Ratto LRC, Carvalho KHP, Silva HR et al. Avaliação da memória operacional fonológica e impulsividade de usuários de drogas atendidos em um centro de atenção integrada à saúde mental. Rev CEFAC. 2012;14: 438-47.

16.Monteiro CFS, Araújo TME, Sousa CMM, Martins MCC, Silva LLL. Adolescentes e o uso de drogas ilícitas: um estudo transversal. Rev enferm UERJ. 2012; 20: 344-8.

17.Brusamarello T. Maftum MA, Mazza VA, Silva AG, Silva TL, Oliveira VC. Papel da família e da escola na prevenção do uso de drogas. Cienc Cuid Saude. 2010; 9:766-73.

18.Bourguignon LN, Guimarães ES, Siqueira MM. A atuação do enfermeiro nos grupos terapêuticos dos Caps ad do Estado do Espírito Santo. Cogitare Enferm. 2010;15: 467-73. 